\title{
Sistema Financiero Popular y Solidario y su evolución desde la implementación de la nueva Ley Orgánica de Economía Popular y Solidaria en el Ecuador
}

\author{
Popular and Solidary Financial System and its Evolution \\ since the Implementation of the New Organic Law of \\ Popular and Solidarity Economy in Ecuador \\ Jaime Roder Ortega-Pereira ${ }^{1}$, Fernando Marcelo Borja-Borja ${ }^{2}$, Liliana \\ Elizabeth Aguilar-Rodríguez ${ }^{3}$, William Marcelo Moreno-Cazar $\square^{4}$
}

\footnotetext{
${ }^{1}$ Universidad Tecnológica Equinoccial (UTE), Quito, Ecuador $\mathbb{D}$ ${ }^{2}$ Universidad Tecnológica Equinoccial (UTE), Quito, Ecuador (ID ${ }^{3}$ Universidad Tecnológica Equinoccial (UTE), Quito, Ecuador (DD

${ }^{4}$ Universidad Tecnológica Equinoccial (UTE), Quito, Ecuador (1D
}

\footnotetext{
william.moreno@ute.edu.ec

Recibido: 14 de marzo del $2017 \quad$ Aprobado: 18 de julio del $2017 \quad$ Disponible en línea: 14 de enero del 2018

Cómo citar este artículo: Ortega-Pereira, J. R., Borja-Borja, F. M., Aguilar-Rodríguez, I. E. y Moreno-Cazar, W. M. (2018).

Sistema Financiero Popular y Solidario y su evolución desde la implementación de la nueva Ley Orgánica de Economía Popular y Solidaria en el Ecuador. Cooperativismo \& Desarrollo, 112(25), 66-77. doi: https://doi.org/10.16925/co.v25i112.2036
}

\section{Resumen}

Introducción: el presente artículo analiza la evolución de las principales cifras de las cooperativas de ahorro y crédito (COAC) que estaban bajo el control de la Dirección Nacional de Cooperativas (DNC) y el Ministerio de Inclusión Económica y Social (MIES), así como de las cooperativas que pertenecían a la Superintendencia de Bancos (SB), y que, posteriormente, pasaron a formar parte del Sistema Financiero Popular y Solidario (SFPS) en los primeros años de vigencia de la Ley Orgánica de Economía Popular y Solidaria (Loeps), hoy bajo el control de la Superintendencia de Economía Popular y Solidaria (SEPS).

Metodología: se aplicó un análisis financiero de variaciones y tendencias con el fin de determinar el crecimiento del SFPS después de la aplicación de la Loeps.

Resultados: las cooperativas de ahorro y crédito han consolidado su participación en el mercado, al pasar de representar un $17,65 \%$ a un $20,20 \%$ de todo el sistema financiero nacional en el periodo analizado.

Conclusiones: pese al efecto sistémico adverso, las cooperativas tuvieron un crecimiento anual compuesto promedio de 8,98 \%, una cifra sustancialmente superior al crecimiento de todo el Sistema Financiero Nacional (SFN) del 1,86 \%, y de los bancos en un 0,20 \% en este mismo periodo.

Palabras clave: bancos, cooperativas de ahorro y crédito, economía popular, economía solidaria, sistema financiero. 


\title{
Popular and Solidary Financial System and its Evolution since the Implementation of the New Organic Law of Popular and Solidarity Economy in Ecuador
}

\begin{abstract}
Introduction: the present article analyzes the evolution of the main data of the credit and savings cooperatives (cscs) that were under the control of the National Cooperatives Directorate (NCD) and the Ministry of Economic and Social Inclusion (MESI), as well as the main data of the cooperatives that belonged to the Superintendency of Banks (SB) and which later became part of the Popular and Solidary Financial System (PSFS) in the first years of the Organic Law of Popular and Solidarity Economy (OLPSE). All of these are under the control of the Superintendency of Popular and Solidarity Economy (SPSE).

Methodology: A financial analysis of variations and trends was applied to determine the PSFs growth after the application of the OLPSE.

Results: Credit and savings cooperatives have consolidated their participation in the market, passing from representing $17.65 \%$ to $20.20 \%$ of the entire national financial system in the analyzed period.

Conclusions: Despite the systemic adverse effect, the cooperatives had an average compound annual growth of $8.98 \%$, a figure substantially higher than the growth of the entire National Financial System (NFS) of $1.86 \%$, and of the banks in a $0.20 \%$ in this same period.
\end{abstract}

Keywords: banks, credit and savings cooperatives, popular economy, solidarity economy, financial system.

\section{Sistema Financeiro Popular e Solidário e sua evolução desde a implantação da nova Lei Orgânica de Economia Popular e Solidária no Equador}

\begin{abstract}
Resumo
Introdução: o presente artigo analisa a evolução das principais cifras das cooperativas de poupança e crédito (COACS, por sua sigla em espanhol) que estavam sob o controle da Direção Nacional de Cooperativas (DNC) e o Ministério de Inclusão Econômica e Social (MIES), assim como das cooperativas que pertenciam à Superintendência de Bancos (SB) e que, posteriormente, passaram a formar parte do Sistema Financeiro Popular e Solidário (SFPS) nos primeiros anos de vigência da Lei Orgânica de Economia Popular e Solidária (Loeps), e que se encontra sob o controle da Superintendência de Economia Popular e Solidária (SEPS).

Metodologia: foi aplicada uma análise financeira de variações e tendências para determinar o crescimento do SFPS depois da aplicação da Loeps.

Resultados: as cooperativas de poupança e crédito consolidaram a sua participação no mercado, passando de $17,65 \%$ a $20,20 \%$ o número de representações de todo 0 sistema financeiro nacional no período analisado.

Conclusões: apesar do efeito sistêmico adverso, as cooperativas tiveram um crescimento anual composto médio de 8,98\%, uma cifra substancialmente superior ao crescimento de todo 0 Sistema Financeiro Nacional (SFN), de 1,86\%, e dos bancos em 0,20\%, neste mesmo período.
\end{abstract}

Palavra-chave: bancos, cooperativas de poupança e crédito, economia popular, economia solidária, sistema financeiro. 


\section{Propósito}

Debido al actual enfoque del Gobierno ecuatoriano de impulsar a las organizaciones que conforman la economía popular y solidaria (EPS), se dio origen a la nueva Ley Orgánica de Economía Popular y Solidaria (Loeps), la cual contempla varios retos para las cooperativas que sustentarán un adecuado manejo de las cifras financieras y económicas que están relacionadas con el negocio y su crecimiento sostenido.

Por tanto, las cooperativas de ahorro y crédito (COAC) que estaban bajo supervisión de la Dirección Nacional de Cooperativas (DNC) y el Ministerio de Inclusión Económica y Social (MIES), así como aquellas que controlaba la Superintendencia de Bancos (sB), se integraron al sector financiero popular y solidario (SFPs), de acuerdo con la Loeps.

El SFPS es normado y regulado por la Superintendencia de Economía Popular y Solidaria (SEPS), amparada en el artículo 147 de la Loeps, que consta en el Registro Oficial 444 de 10 de mayo de 2011, y su Reglamento a la Ley Orgánica de Economía Popular y Solidaría (RLOEPS).

Este artículo pretende analizar el crecimiento del sfrs después de la aplicación de la Ley y la creación de la nueva Superintendencia.

\section{Descripción}

Es importante definir que dentro de la Loeps existen tres tipos de organizaciones: 1) sector comunitario, 2) sector asociativo y 3) sector cooperativo.

En la Loeps, el artículo 15 define el sector comunitario como el conjunto de organizaciones vinculadas por relaciones de territorio, familiares, identidades étnicas, culturales, de género, de cuidado de la naturaleza, urbanas o rurales; o bien de comunas, comunidades, pueblos y nacionalidades que, mediante el trabajo conjunto tienen por objeto la producción, la comercialización, la distribución y el consumo de bienes o servicios lícitos y socialmente necesarios, de forma solidaria y autogestionada, bajo los principios de la ley (Loeps, p. 6).

En el artículo 18, el sector asociativo es el conjunto de asociaciones constituidas por personas naturales con actividades económicas productivas similares o complementarias con el objeto de producir, comercializar y consumir bienes y servicios lícitos y socialmente necesarios, autoabastecerse de materia prima, insumos, herramientas, tecnología, equipos y otros bienes, o bien comercializar su producción de forma solidaria y autogestionada bajo los principios de la ley (Loeps, p. 6).

En el artículo 21, el sector cooperativo aparece como el conjunto de cooperativas entendidas como sociedades de personas que se han unido de forma voluntaria a fin de satisfacer sus necesidades económicas, sociales y culturales en común, mediante una empresa de propiedad conjunta y de gestión democrática con personalidad jurídica de derecho privado e interés social. Las cooperativas, en su actividad y relaciones, se sujetarán a los principios establecidos en la Ley Orgánica de Economía Popular y Solidaria y del sector financiero popular y solidario, a los valores y principios universales del cooperativismo y a las prácticas de buen gobierno corporativo (Loeps, p. 7).

Dentro del sector cooperativo, se encuentran las cooperativas no financieras y las cooperativas financieras; estas últimas pertenecen al SFPS y se denominan "COAC".

Al mes de abril del 2013, existían 3932 cooperativas, concentradas en organizaciones tales como cooperativas de ahorro y crédito, transporte, vivienda, producción, consumo y otros servicios, según datos de la SEPS. La SEPS, con base en la información de la DNC y el Servicio de Rentas Internas (SRI), señala:

Las cooperativas de ahorro y crédito (COAC) constituyen el grupo de mayor tamaño en términos de activos, pasivos y patrimonio, respecto del total nacional de cooperativas. Según estimaciones, a diciembre de 2012, los activos de las COAC ascendieron a us 5.795,8 millones, lo que representa el $93 \%$ del total del sector cooperativo. (sEPs, 2013, p. 4)

El sector financiero popular y solidario lo conforman las cooperativas de ahorro y crédito (COAC) que operan en el país y adecuaron sus estatutos en la Superintendencia de Economía Popular y Solidaria, de acuerdo con la "Disposición transitoria primera" de la Ley Orgánica de Economía Popular y Solidaria. Entre las atribuciones legales de la SEPS, de acuerdo con el artículo 147, literal f de la Loeps, se menciona literalmente "levantar estadísticas de las actividades que realizan las organizaciones sujetas a esta Ley". Adicionalmente, el artículo 101 de la Loeps menciona que las cooperativas deben clasificarse en segmentos con el fin de emitir políticas y 
regulaciones específicas. Con estos antecedentes, la sEPs pone a disposición de la ciudadanía el presente boletín financiero que consolida la información contable - con corte al 30 de junio del 2013 - de todas las cooperativas de ahorro y crédito que enviaron su información a la Superintendencia de Economía Popular y Solidaria (SEPS, 2013).

Según Richer (2001), "la cooperativa es una asociación autónoma de personas voluntariamente unidas para satisfacer sus aspiraciones y necesidades económicas, sociales y culturales comunes por medio de una empresa cuya propiedad es colectiva y en la cual el poder es ejercido democráticamente" (p. 13). Por tanto, en una cooperativa se ejerce la democracia por medio de votación (una persona un voto), a diferencia de las corporaciones o los bancos donde una acción equivale a un voto y, por tanto, se aplica la regla del oro: "Quien tiene el oro, pone las reglas". Este es quizá el punto fuerte de las COAC y, a su vez, su punto más débil.

De acuerdo con González, Arias y San Bartolomé (2012), las cooperativas surgen de la propia comunidad a fin de satisfacer las necesidades de sus socios, así como las de la propia comunidad.

Las COAC, de acuerdo con la resolución JR-STE-2012-003 de la Junta de Regulación, se clasificaban en cuatro segmentos.

En esta clasificación, a las 40 cooperativas que estaban controladas por la sB las agruparon en el Segmento 4 (s4), mientras que las cooperativas controladas por la DNC y el MIEs se agruparon en el Segmento 1 (s1), Segmento 2 (s2) y Segmento 3 (s3); según las variables: número de socios, valor de los activos y número de cantones en los que operan (tabla 1).

En el boletín financiero, la sEPs solo consideró 773 cooperativas que enviaron la información de las 947 censadas a junio del 2013 (tabla 2).
Tabla 2. Cooperativas consideradas en el Boletín financiero de la sEPS

\begin{tabular}{ll}
\hline Segmentos & \multicolumn{1}{c}{ N. $^{\circ}$ Cooperativas } \\
\hline Segmento 1: & $\begin{array}{l}25 \text { cooperativas, de un total de } 25 \text { coope- } \\
\text { rativas }\end{array}$ \\
Segmento 2: & $\begin{array}{l}34 \text { cooperativas, de un total de } 35 \text { coope- } \\
\text { rativas }\end{array}$ \\
Segmento 3: & $\begin{array}{l}85 \text { cooperativas, de un toral de } 85 \text { coope- } \\
\text { rativas }\end{array}$ \\
Segmento 4: & $\begin{array}{l}163 \text { cooperativas, de un total de } 185 \\
\text { cooperativas }\end{array}$ \\
Segmento 5: & $\begin{array}{l}322 \text { cooperativas, de un total de } 463 \\
\text { cooperativas }\end{array}$ \\
Sin segmen- & $\begin{array}{l}0 \text { cooperativas, de un total de } 7 \text { coopera- } \\
\text { tivas }\end{array}$ \\
to: & $\begin{array}{l}\text { 629 cooperativas, de un total de } 802 \\
\text { cooperativas }\end{array}$ \\
Total: &
\end{tabular}

Nota. Tomada del Boletín financiero, por la Superintendencia de Economía Popular y Solidaria-SEPs, 2013, junio 30.

Las 773 cooperativas que iniciaron mantenían más de usD 6000 millones en activos como se puede observar en la tabla 3. El activo más importante de las COAC es la cartera de crédito, la misma que representa USD 4792 millones (tabla 3).

Tabla 3. Activos de las COAC del sfrs (miles de USD)

\begin{tabular}{llll}
\hline \multicolumn{1}{c}{ Activos } & & N. $^{\circ}$ & \multicolumn{1}{c}{ Jun-13 } \\
\hline Segmento 1 & 359 & 94841 \\
Segmento 2 & 296 & 609470 \\
Segmento 3 & 78 & 1538966 \\
Segmento 4 & 40 & 4208088 \\
Total & 773 & 6451365 \\
\hline
\end{tabular}

Nota. Tomada del Boletín financiero, por la Superintendencia de Economía Popular y Solidaria-sePs, 2013, junio 30.

Tabla 1. Segmentación de las cooperativas DNC y MIES

\begin{tabular}{cccc}
\hline Segmento & Activos & Cantones & Socios \\
\hline Segmento 1 & $0-250000,00$ & 1 & más de 700 \\
Segmento 1 & $0-1000000,00$ & 1 & hasta 700 \\
Segmento 2 & $250000,01-1000000,00$ & 1 & más de 700 \\
Segmento 2 & $1100000,00-9600000,00$ & 2 o más & Sin importar el número de socios \\
Segmento 2 & 1100000 o más & Sin importar el múmero cantones que opera & hasta 7100 \\
Segmento 3 & 9600000,00 o más & Sin importar el múmero cantones que opera & más de 7100 \\
Segmento 3 & & Sin importar el múmero cantones que opera & hasta 7100 \\
\hline
\end{tabular}

Nota. Tomada del Boletín financiero, por la Superintendencia de Economía Popular y Solidaria-SEPs, 2013, junio 30. 
La entrega de usD 4792 millones en cartera de crédito significaría que, proporcionalmente, se está otorgando por parte de este sector más crédito con respecto a sus captaciones, debido a que el ahorro de las COAC alcanza los USD 4651 millones a junio del 2013 (tablas 4 y 5).

Tabla 4. Cartera de las COAC del sFPs (miles de USD)

\begin{tabular}{lcc}
\hline \multicolumn{1}{c}{ Cartera } & N. $^{\circ}$ & Jun-13 \\
\hline Segmento 1 & 359 & 68309 \\
Segmento 2 & 296 & 445703 \\
Segmento 3 & 78 & 1154437 \\
Segmento 4 & 40 & 3123757 \\
Total & 773 & 4792206 \\
\hline
\end{tabular}

Nota. Tomada del Boletín financiero, por la Superintendencia de Economía Popular y Solidaria-sePs, 2013, junio 30.

Tabla 5. Captaciones de las COAC del sFPs (miles de USD)

\begin{tabular}{lcc}
\hline $\begin{array}{c}\text { Obligaciones con } \\
\text { el Público }\end{array}$ & N. ${ }^{\circ}$ & Jun-13 \\
\hline Segmento 1 & 359 & 59505 \\
Segmento 2 & 296 & 404174 \\
Segmento 3 & 78 & 1054217 \\
Segmento 4 & 40 & 3133220 \\
Total & 773 & 4651116 \\
\hline
\end{tabular}

Nota. Tomada del Boletín financiero, por la Superintendencia de Economía Popular y Solidaria-sEPs, 2013, junio 30.

Las cooperativas mantienen presencia a nivel nacional, por tanto, su impacto afecta a gran parte de la población: "En cuanto a los socios, a diciembre del 2012, el número total de socios declarados por las cooperativas de ahorro y crédito a nivel nacional bordea los 4,6 millones de personas" (sEPs, 2013, p. 4). En España, la expansión territorial de las instituciones del sistema financiero se ha realizado principalmente por la apertura de nuevas oficinas y la diversificación de productos financieros (Palomo y Mateu, 1999).

En el ámbito financiero, al seleccionar las cinco COAC con mayor volumen de colocación en el mercado, estas mantienen una cartera total conjunta a junio del 2012 de usD 1142 millones, monto que es equivalente al saldo de cartera total a la misma fecha de 14 bancos privados medianos y pequeños (sEPS, 2012). Esto convierte a este sector en el segundo mayor competidor de productos financieros después de los bancos.
Con la implementación de la Loeps, las COAC -que estaban bajo el control de la DNC y del MIEsadoptaron la estructura de los estados financieros bajo el Catalogo Único de Cuentas (CUC), ${ }^{1}$ utilizado obligatoriamente por todas las instituciones que conforman el Sistema Financiero Nacional (SFN) y las COAC; esto proporcionó una estandarización de la información con la que trabajarán a futuro estas organizaciones.

A diciembre del 2013, las 773 coAc que iniciaron el SFPS sumadas representaban más de USD 7107 millones en activos, con una participación de mercado del 17,65 \% de todo el Sistema Financiero Nacional (tabla 6). ${ }^{2}$

Tabla 6. Activos del SFN (miles de USD)

\begin{tabular}{lcc}
\hline Activos & Dec-13 & Dec-13 \\
\hline Bancos & 30738407 & $76,32 \%$ \\
Cooperativas & 7107301 & $17,65 \%$ \\
Sociedades & 1790170 & $4,44 \%$ \\
Financieras & 638485 & $1,59 \%$ \\
Mutualistas & 40274362 & $100,00 \%$ \\
Total & \\
\hline
\end{tabular}

Nota. Tomada de sB y sePs

Las 773 cooperativas han otorgado, a diciembre del 2013, el 22,30\% de los créditos de todo el SFN en los cuatro tipos de créditos existentes a esa fecha (tabla 7).

Tabla 7. Cartera de créditos del SFN (miles de USD)

\begin{tabular}{lcc}
\hline \multicolumn{1}{c}{ Cartera } & Dec-13 & Dec-13 \\
\hline Bancos & 17257688 & $70,26 \%$ \\
Cooperativas & 5478730 & $22,30 \%$ \\
Sociedades & 1410860 & $5,74 \%$ \\
Financieras & 416611 & $1,70 \%$ \\
Mutualistas & 24563869 & $100,00 \%$ \\
Total & \\
\hline
\end{tabular}

Nota. Tomada de sB y SEPS

1 Las instituciones controladas por la SEPS mantienen un Catálogo de Único de Cuenta (CUC), según Resolución N. ${ }^{\circ}$ SEPSIFPS-2012-0025 de 05 de diciembre de 2012.

2 Datos obtenidos en la Superintendencia de Bancos y Seguros (sBS) y la Superintendencia de Economía Popular y Solidaria (SEPS). 
Estas mismas 773 cooperativas a diciembre del mismo año han recaudado del público el 17,03 \% de los recursos de todo el SFN, lo que demuestra una notable aceptación de estas organizaciones debido a que los socios y clientes confían en ellas y les depositan sus ahorros (tabla 8).

Tabla 8. Obligaciones con el público del SFN (miles de USD)

\begin{tabular}{lll}
\hline Obligaciones con el público & \multicolumn{1}{c}{ Dec-13 } & \multicolumn{1}{c}{ Dec-13 } \\
\hline Bancos & 24886200 & $79,90 \%$ \\
Cooperativas & 5304836 & $17,03 \%$ \\
Sociedades Financieras & 953978 & $3,06 \%$ \\
Mutualistas & 538485 & $1,73 \%$ \\
& 31145014 & $100,00 \%$ \\
\hline
\end{tabular}

Nota. Tomada de sB y SEPS

Con esas cifras de participación de mercado, se hace necesario realizar un seguimiento que sirva para la administración y la toma de decisiones, en el que se deben aplicar herramientas de análisis de resultados, de tendencias y proyecciones. En este sentido, Horngren, Foster y Datar (2002) sostienen que "un sistema de control de la administración es un medio de recolectar y utilizar información para ayudar y coordinar el proceso de planeación y de toma de decisiones de control" (p. 788). Lo que se puede aplicar al análisis de sectores, como es el caso de las COAC.

Los análisis de resultados pueden y deben ser cuantificados, tanto en el mundo de los negocios como en el análisis estratégico. De acuerdo con Gareth y George (2003), el análisis de resultados es una metodología que permite a las compañías obtener una visión de sus resultados por cada periodo que puede ser mensual, bimensual, trimestral, semestral o anual. Meigs (1980), por su parte, propone que analizar implica el proceso de identificación y clasificación para el ulterior estudio de todos los componentes internos y externos que involucra la toma de decisiones.

\section{Punto de vista}

Se ha utilizado un análisis financiero de variaciones y tendencias para determinar el crecimiento del sfps después de la aplicación de la Loeps, de acuerdo con lo planteado por Ross, Westerfield y Jordan (2001) en la ecuación 1.

$$
\begin{aligned}
& V=X_{1}-X_{0} \\
& \text { Donde: } \\
& V=\text { es la variación absoluta } \\
& X_{1}=\text { Observación año actual } \\
& X_{0}=\text { Observación año anterior }
\end{aligned}
$$

Otra metodología utilizada para determinar el crecimiento del SFPS fue la tasa de crecimiento, la cual se aplicó a partir de la fórmula del interés compuesto, de modo que se estableció una tasa anual de crecimiento del SFPS, como lo muestra Portus (1997) en la ecuación 2.

$$
i=\left\lceil\frac{F}{A}\right\rceil^{\frac{1}{n}}-1
$$

Donde:

$\mathrm{i}=$ es la tasa de interés compuesta

$F=$ Valor futuro

$A=$ Valor actual

$n=$ número de periodos

Del análisis de la evolución realizado al SFPS, desde la promulgación de la Loeps y su RLOEPS, se obtuvieron los siguientes resultados.

\section{Segmentación de las COAC}

El criterio de la segmentación ha cambiado con el tiempo. De los datos obtenidos de la SEPS al 2015, presenta modificaciones conforme a lo dispuesto en el artículo 14, numeral 35 del Código Orgánico Monetario y Financiero, en el que se determina, entre las funciones de la Junta de Política y Regulación Monetaria y Financiera, "establecer la segmentación de las entidades del Sector Financiero Popular y Solidario" (p. 8); la Junta de Política y Regulación Monetaria y Financiera expide la resolución N. ${ }^{\circ}$ 038-2015-F el 13 de febrero de 2015, en la que establece en el artículo 1 que las entidades del sector financiero popular y solidario, de acuerdo con el tipo y el saldo de sus activos, se ubicarán en los segmentos que se presentan en la tabla 9 (p. 1).

Se puede observar que el segmento con mayor presencia de cooperativas es el Segmento 5 (s5), con 322 organizaciones que reportan de un total de 463 censadas.

Mientras que el s1 cuenta con 25 cooperativas, 24 de ahorro y crédito de primer piso y Financoop como cooperativa de segundo piso, en este segmento se ubican las cooperativas más grandes con activos 
Tabla 9. La segmentación de las entidades del sector financiero popular y solidario

\begin{tabular}{cl}
\hline Segmento & \multicolumn{1}{c}{ Activos (USD) } \\
\hline 1 & Mayor de 80000000,00 \\
2 & Mayor de 20000000,00 hasta 80000000,00 \\
3 & Mayor de 5000000,00 hasta 20000000,00 \\
4 & Mayor de 1000000,00 hasta 5000000,00 \\
5 & $\begin{array}{l}\text { Hasta } 1000000,00 \text { Cajas de ahorro, bancos } \\
\text { comunales y cajas comunales }\end{array}$ \\
\hline
\end{tabular}

Nota. Tomada del Boletín financiero, por la Superintendencia de Economía Popular y Solidaria-sEPs, 2013, junio 30.

Tabla 10. Número de organizaciones por segmento de las entidades del sector financiero popular y solidario

\begin{tabular}{ll}
\hline \multicolumn{1}{c}{ Segmentos } & \multicolumn{1}{c}{$\mathrm{N}^{\circ}$ Cooperativas } \\
\hline Segmento 1: & $\begin{array}{l}25 \text { cooperativas, de un total de } 25 \mathrm{coo}^{-} \\
\text {perativas }\end{array}$ \\
Segmento 2: & $\begin{array}{l}34 \text { cooperativas, de un total de } 35 \mathrm{coo}^{-} \\
\text {perativas }\end{array}$ \\
Segmento 3: & $\begin{array}{l}85 \text { cooperativas, de un toral de } 85 \text { coo- } \\
\text { perativas }\end{array}$ \\
Segmento 4: & $\begin{array}{l}163 \text { cooperativas, de un total de } 185 \\
\text { cooperativas }\end{array}$ \\
Segmento 5: & $\begin{array}{l}322 \text { cooperativas, de un total de } 463 \\
\text { cooperativas }\end{array}$ \\
Sin segmento: & $\begin{array}{l}\text { Cero cooperativas, de un total de siete } \\
\text { cooperativas }\end{array}$ \\
Total: & $\begin{array}{l}\text { 629 cooperativas, de un total de } 802 \\
\text { cooperativas }\end{array}$ \\
\hline
\end{tabular}

Nota. Tomada del Boletín financiero, por la Superintendencia de Economía Popular y Solidaria-SEPs, 2013, junio 30.

superiores a los USD 80 millones, mientras que en el s2 se ubican las COAC que van de USD 20 a 80 millones, con 34 integrantes que reportan información. Parte de estas, junto con las del actual s1, formaban antes el antiguo $s 4$ (hasta febrero del 2015).

\section{Evolución de los activos y la cartera de crédito}

El cambio efectuado por las SEPs de cuatro a cinco segmentos obedece a una nueva forma de clasificación, en la que se asume como única variable el monto de los activos. Si se realiza un comparativo entre todas las organizaciones que forman parte del mercado de dinero - también conocido como
SFN-, este se compone de instituciones financieras, tales como bancos privados, mutualistas, sociedades financieras y entre ellas las COAC, las cuales en su conjunto manejan USD 41782 millones a diciembre del 2015. Así, entonces, se observa que el 20,20\% del mercado pertenece a las COAC, lo que las posiciona como actores importantes del SFN (tabla 11).

Tabla 11. Participacion de las COAC del SFN (miles de USD)

\begin{tabular}{lcc}
\hline \multicolumn{1}{c}{ Activos } & Dec-15 & $\%$ \\
\hline Bancos & 308640 & $76,32 \%$ \\
Cooperativas & 7107301 & $17,65 \%$ \\
Sociedades Financieras & 1790170 & $4,44 \%$ \\
Mutualistas & 638485 & $1,59 \%$ \\
Total & 40274362 & $100,00 \%$ \\
\hline
\end{tabular}

Nota. Tomada de sB y sePs

La evolución de estos primeros años de la aplicación de la ley ha sido favorable para las COAC, pese a que existe una tendencia decreciente si se considera la variación positiva en el 2014, con cerca de USD 1000 millones en sus activos, comparado con el 2015 cuando tan solo creció usd 380 millones (tabla 12). Esto significó un incremento anual compuesto promedio en este periodo del $8,98 \%$, lo que contrasta con el crecimiento del SFN, tan solo del 1,86\%, mientras que los bancos privados crecieron en un $0,20 \%$ en este mismo periodo. Lo anterior ocasionó un incremento importante en el sector cooperativo pese al efecto sistémico, al pasar de USD 7107 a USD 8440 millones en activos.

Tabla 12. Evolución de las COAC del sector financiero popular y solidario (miles de USD)

\begin{tabular}{lccc}
\hline \multicolumn{1}{c}{ Cooperativas } & Dec-13 & Dec-14 & Dec-15 \\
\hline Activo & 7107301 & 8061795 & 8440322 \\
Pasivo & 6059176 & 6873715 & 7090406 \\
Patrimonio & 1048125 & 1188080 & 1349916 \\
Resultados & 74231 & 75549 & 78451 \\
Variación Activo & & 954494 & 378527 \\
Variación Pasivo & & 814540 & 216691 \\
Variación & & 139954 & 161836 \\
Patrimonio & & & \\
Variación & & 139954 & 161836 \\
\hline
\end{tabular}

Nota. Tomada del Boletín financiero, por la Superintendencia de Economía Popular y Solidaria-sEPs, 2013, junio 30. 
El sFps ha sufrido disminuciones del número de participantes. A partir de la presente Ley, han pasado de un total de 773 cooperativas que reportaban en febrero del 2013, a 629 organizaciones en el 2015 (tabla 13). Esta reducción se ha originado por la fusión o absorción de algunas de ellas como una medida para su fortalecimiento, así como la transformación en bancos, como es el caso de la Cooperativa Nacional y Codesarrollo. Por otra parte, han ingresado al control nuevas cooperativas como Muschu Runa, Policía Nacional, Servidores Públicos del Ministerio de Educación y Cultura, así como la Cooperativa Pilahuin Tío, según datos obtenidos de la SEPS (2012).

Tabla 13. Evolución de las coAc del s1 (miles de usD)

\begin{tabular}{lccc}
\hline \multicolumn{1}{c}{ Cooperativas } & Dec-13 & Dec-14 & Dec-15 \\
\hline Activo & 4542816 & 5169033 & 5441881 \\
Pasivo & 3904637 & 4452436 & 4613632 \\
Patrimonio & 638179 & 716597 & 828249 \\
Resultados & 54620 & 59076 & 64438 \\
Variación Activo & & 626217 & 272848 \\
Variación Pasivo & & 547799 & 161196 \\
Variación Patri- & & 78417 & 111652 \\
monio & & 78417 & 111652 \\
Variación & & &
\end{tabular}

Nota. Tomada del Boletín financiero, por la Superintendencia de Economía Popular y Solidaria-sEPs, 2013, junio 30.

Una agrupación importante de estas cooperativas son las que conforman el s1 y representan el $64,47 \%$ de los activos a diciembre del 2015 de todo el SFPS, pese a que este segmento también ha sufrido la reducción del número de organizaciones, puesto que, en el 2013, le pertenecían 40 COAC y, a diciembre del 2015, solo existían 24 cooperativas en las que se encuentran las COAC: Juventud Ecuatoriana Progresista, Jardín Azuayo, Policía Nacional, 29 de Octubre y Cooprogreso, de modo que se logra una participación del 46,59 \% de los activos del s1, y crece con una tasa compuesta del 9,45\% superior al registrado por el SFPS.

Las COAC del SFPs tienen el 24,52 \% de la participación de mercado de la cartera bruta, la misma que se ubica en consumo y microcrédito, fundamentalmente.

El activo más importante y la razón de ser de una institución financiera es la cartera de crédito, la misma que representa el 79,18 \% de los activos
Tabla 14. Participación de la cartera bruta (miles de USD)

\begin{tabular}{lcc}
\hline \multicolumn{1}{c}{ Cartera bruta } & Dec-15 & $\%$ \\
\hline Bancos & 18773251 & $68,88 \%$ \\
Cooperativas & 6683403 & $24,52 \%$ \\
Sociedades & 1357515 & $4,98 \%$ \\
Financieras & 440118 & $1,61 \%$ \\
Mutualistas & 27254297 & $100,00 \%$ \\
Total & & \\
\hline
\end{tabular}

Nota. Tomada de sB y SEPS

del SFPs, de lo cual el saldo de la cartera ha destinado el $49,03 \%$ a consumo, seguido por un $41,40 \%$ en microcrédito, $5,63 \%$ a crédito inmobiliario y el $3,32 \%$ a crédito comercial.

De los activos del s1, la cartera de crédito representa el 80,27 \%, de lo cual el saldo de esta se ha destinado en un $53,18 \%$ a consumo, seguido por un $35,82 \%$ en microcrédito, el $6,48 \%$ a crédito inmobiliario y un $4,12 \%$ a crédito comercial.

La cartera bruta del s1, en cambio, representa el $65,36 \%$ del total del sFPs con un crecimiento del $10,84 \%$ compuesto anual, de modo que son las más colocadoras las COAC: Juventud Ecuatoriana Progresista, Jardín Azuayo, Policía Nacional, 29 de Octubre y Cooprogreso, que representan el 46,15\% de la cartera de este segmento y el 30,16 \% del sFPs.

\section{Evolución de los pasivos y las obligaciones con el público}

En cuanto a los pasivos de todas las organizaciones que son parte del sFPs representan el 19,31 \% del SFN, y estos presentan un crecimiento del $8,18 \%$ compuesto anual en este mismo periodo. Por su parte, el SFN en su conjunto creció en un 1,04\%, lo que contrasta con la disminución del 0,47 \% que sufrieron los bancos privados a causa de la disminución de las cuentas de obligaciones con el público por más usd 3181 millones, de manera que fueron los mayores afectados.

El pasivo más importante está relacionado con las obligaciones con el público, las cuales representan el 90,20 \% del financiamiento de terceros, el cual está divido a su vez en dos grandes grupos; 1 ) el saldo de los depósitos a plazos que representa el 60,21 \% y 2) el que conforman los depósitos a la vista, las operaciones de reporto, depósitos en garantía y depósitos restringidos, que representan el 39,79\% de las obligaciones con el público. 
Tabla 15. Participación de las COAC del SFN (miles de USD)

\begin{tabular}{lcc}
\hline \multicolumn{1}{c}{ Pasivo } & Dec-15 & $\%$ \\
\hline Bancos & 27567603 & $75,07 \%$ \\
Cooperativas & 7090406 & $19,31 \%$ \\
Sociedades finan- & 1456231 & $3,97 \%$ \\
cieras & 608079 & $1,66 \%$ \\
Mutualistas & 36722320 & $100,00 \%$ \\
Total IFIs & \\
\hline
\end{tabular}

Nota. Tomada del Boletín financiero, por la Superintendencia de Economía Popular y Solidaria-seps, 2013, junio 30.

Los pasivos del s1 representan el 65,07 \% de los pasivos de todo el SFPS a diciembre del 2015 , con un crecimiento compuesto del $8,70 \%$, pese a que se han reducido el número de organizaciones. A diciembre del 2015, contaban con solo 24 cooperativas en este segmento, en las que se encuentran a las COAC: Juventud Ecuatoriana Progresista, Jardín Azuayo, Cooprogreso, Policía Nacional y 29 de Octubre, que representan el 47,34\% del s1. El pasivo más importante de este segmento lo representan los depósitos a plazo con la participación del $61,38 \%$, mientras que el 38,62 \% lo componen los depósitos a la vista, operaciones de reporto, depósitos en garantía y depósitos restringidos.

\section{Evolución de los depósitos de plazos (DPF)}

Las COAC del sFPs representan el 30,71 \% de la participación de mercado y de las captaciones en depósitos a plazo fijo (DPF), lo cual es una consecuencia de la confianza demostrada por sus socios y clientes (tabla 16).

Tabla 16. Participación de los depósitos a plazos (miles de UsD)

\begin{tabular}{lcc}
\hline \multicolumn{1}{c}{ Depósitos a Plazos } & Dec-15 & $\%$ \\
\hline Bancos & 7402148 & 59,045 \\
Cooperativas & 3850462 & $30,71 \%$ \\
Sociedades financieras & 955601 & $7,62 \%$ \\
Mutualistas & 328381 & $2,62 \%$ \\
Total IFIs & 12536601 & $100,00 \%$ \\
\hline
\end{tabular}

Nota. Tomada de sB y sePs

Los depósitos a plazos del s1 representan el $67,86 \%$ del total del sFPs con un crecimiento del $14,49 \%$ compuesto anual, y son las más captadoras las COAC: Juventud Ecuatoriana Progresista, Jardín Azuayo, Cooprogreso, "29 de octubre" y Policía Nacional (representan el 47,61 \% de este segmento).

\section{Evolución de los depósitos a la vista}

Las cooperativas de ahorro y crédito cOAC del SFPS representan el 12,85 \% de la participación de mercado de los depósitos a la vista del sFN (tabla 17).

Tabla 17. Participación de los depósitos a la vista (miles de USD)

\begin{tabular}{lcc}
\hline Depósticos a la Vista & Dec-15 & $\%$ \\
\hline Bancos & 17011027 & $85,87 \%$ \\
Cooperativas & 2545009 & $12,85 \%$ \\
$\begin{array}{l}\text { Sociedades Finan- } \\
\text { cieras }\end{array}$ & 12482 & $0,06 \%$ \\
Mutualistas & 242390 & $1,22 \%$ \\
Total & 19810907 & $100,00 \%$ \\
\hline
\end{tabular}

Nota. Tomada de sB y sEPs

Los depósitos a la vista del s1 representan el $66,56 \%$ del total del sfps, con un crecimiento de apenas un 7,09 \% compuesto anual, y son las más captadoras las COAC: Juventud Ecuatoriana Progresista, Jardín Azuayo, 29 de octubre, Policía Nacional y Riobamba (representan el 45,34 \% del segmento).

\section{Evolución del patrimonio}

El patrimonio de las COAC representa el 26,68 \% del SFN, el cual se conforma por la cuenta de certificados de aportación que le dan la categoría de socio, además de las cuentas de reservas y utilidades (tabla 18). El patrimonio presenta un crecimiento de $13,49 \%$ compuesto anual en este mismo periodo, lo que compensa de esta manera el menor crecimiento de los pasivos y sus obligaciones con el público con respecto a los activos, impulsados por los buenos resultados obtenidos. Así, el capital social representa el $50,86 \%$ y las reservas el $37,70 \%$.

Tabla 18. Participación del patrimonio (miles de USD)

\begin{tabular}{lcc}
\hline \multicolumn{1}{c}{ Patrimonio } & Dec-15 & $\%$ \\
\hline Bancos & 3296476 & $65,14 \%$ \\
Cooperativas & 1349916 & $26,68 \%$ \\
Sociedades financieras & 334958 & $6,62 \%$ \\
Mutualistas & 79152 & $1,56 \%$ \\
Total & 5060502 & $100,00 \%$ \\
\hline
\end{tabular}

Nota. Tomada de sB y sePs 
En el s1, el patrimonio representa el $61,36 \%$ a diciembre del 2015 de todo este sector, con un crecimiento compuesto del 13,92 \%, en el que son parte las COAC: Juventud Ecuatoriana Progresista, Policía Nacional, Jardín Azuayo, "29 de octubre" y San Francisco (representan el 43,53 \% del segmento).

De esta manera, el capital social recursos aportados por los socios de la cooperativa en certificados de aportación representa el 47,58 \% y el 39,74 \% en reservas, entre cuales la más importante es la reserva legal con el $75,10 \%$.

\section{Evolución de los resultados}

La cuenta de resultados es la diferencia entre las cuentas Cinco y Cuatro del Catálogo Único de Cuentas (CUC). Las organizaciones que son parte del SFPS presentan un modesto crecimiento del $2,80 \%$ compuesto anual en este periodo, que es, sin embargo, el doble del registrado por el SFN (alcanzó el 1,29\%), y los bancos privados (con apenas el $0,56 \%$; tabla 19). No obstante, las COAC representan el 19,97 \% de participación de los resultados obtenidos por el SFN.

Tabla 19. Participación de los resultados (miles de USD)

\begin{tabular}{lcc}
\hline \multicolumn{1}{c}{ Resultados } & Dec-15 & $\%$ \\
\hline Bancos & 271029 & $68,99 \%$ \\
Cooperativas & 78451 & $19,97 \%$ \\
Sociedades Financieras & 40178 & $10,23 \%$ \\
Mutualistas & 3218 & $0,82 \%$ \\
Total & 392876 & $100,00 \%$ \\
\hline
\end{tabular}

Nota. Tomada de sB y SEPs

Los resultados obtenidos por el s1 son más alentadores, pues con un porcentaje del $82,14 \%$ de participación a diciembre del 2015 de todo el SFPS, presentan un crecimiento del 8,62\%. En este segmento se encuentran las coAC: Juventud Ecuatoriana Progresista, Jardín Azuayo, San Francisco, Cacpeco y Alianza del Valle (representan el 51 \%).

Todas las instituciones financieras se crearon, fundamentalmente, a fin de realizar intermediación financiera; por consiguiente, deben captar recursos del público y colocarlos en créditos a los sectores demandantes (tabla 20).

Pese a que las COAC no se diseñaron para generar una ganancia económica, sino más bien un beneficio colectivo. Esto lo contempla Coraggio (2011) al afirmar que la economía es social porque produce sociedad y no solo utilidades económicas (tabla 21).

Tabla 20. Evolución de las cifras del negocio de las COAC del SFPs (miles de USD)

\begin{tabular}{lccc}
\hline \multicolumn{1}{c}{ Cooperativas s1 } & Dec-13 & Dec-14 & Dec-15 \\
\hline Depósitos a plazos & 2937483 & 3548913 & 3850462 \\
Depósitos a la vista & 2367353 & 2627101 & 2545009 \\
Cartera bruta & 5478730 & 6217996 & 6683403 \\
$\begin{array}{l}\text { Variación depósitos } \\
\text { a plazos }\end{array}$ & 611429 & 301550 \\
$\begin{array}{l}\text { Variación depósitos } \\
\text { a la vista }\end{array}$ & & \\
Variación de cartera & & 739266 & 465406 \\
Variación neta & & 131911 & -245949 \\
\hline
\end{tabular}

Nota. Tomada del Boletín financiero, por la Superintendencia de Economía Popular y Solidaria-sEPs, 2013, junio 30.

Tabla 21. Evolución de las cifras del negocio de las COAC s1 de las entidades del sFPs (miles de USD)

\begin{tabular}{lrrr}
\hline \multicolumn{1}{c}{ Cooperativas s1 } & Dec-13 & Dec-14 & Dec-15 \\
\hline Depósitos a plazos & 1993436 & 2434555 & 2613011 \\
Depósitos a la vista & 1477021 & 1646402 & 1693976 \\
Cartera bruta & 3555541 & 4050964 & 4368070 \\
$\begin{array}{l}\text { Variación depósitos } \\
\text { a plazos }\end{array}$ & 441118 & 178457 \\
$\begin{array}{l}\text { Variación depósitos } \\
\text { a la vista }\end{array}$ & & \\
Variación de cartera & & 495381 & 47574 \\
Variación neta & & 115076 & -91075 \\
\hline
\end{tabular}

Nota. Tomada del Boletín financiero, por la Superintendencia de Economía Popular y Solidaria-sePs, 2013, junio 30.

\section{Conclusiones}

Las cooperativas de ahorro y crédito han consolidado su participación de mercado al pasar de un $17,65 \%$ a un 20,20 \% de todo el Sistema Financiero Nacional en el periodo analizado, lo que significa pasar de manejar USD 7107 millones a USD 8440 millones en activos. Así, pese al efecto sistémico adverso, las cooperativas tuvieron un crecimiento anual compuesto promedio del 8,98 \%, lo cual es muy superior en comparación tanto con el crecimiento de todo el SFN (1,86 \%), como de los bancos $(0,20 \%)$ en este mismo periodo.

Los activos de las cooperativas que conforman el actual Segmento 1 (activos > USD 80 millones) 
representan el 64,47 \% a diciembre del 2015 de todos los activos del SFPs, pese a que son tan solo 24 de 629 organizaciones, lo que significa un 3,82 \% del número de cooperativas en operación, con un crecimiento anual compuesto promedio del 9,45\% en este periodo.

Las COAC más grandes son: Juventud Ecuatoriana Progresista, Jardín Azuayo, Policía Nacional, "29 de octubre" y Cooprogreso; estas cinco cooperativas tienen una participación del 46,59 \% de los activos del s1.

La evolución de la cartera de crédito de las cooperativas de ahorro y crédito del Sistema Financiero Popular y Solidario tenían una participación del $22,30 \%$ en el 2013 , y pasan al 24,52 \% de la participación de mercado de la cartera bruta a diciembre del 2015, la cual está colocada en cartera de consumo y microcrédito, fundamentalmente.

La cartera de crédito de las cooperativas del SFPs representa el 79,18 \% de sus activos totales, de los que el saldo se ha destinado en un $49,03 \%$ a cartera de consumo, seguido por un $41,40 \%$ a cartera de microcrédito, a crédito inmobiliario con un $5,63 \%$, y en un $3,32 \%$ a crédito comercial.

De los activos del segmento uno, la cartera de crédito representa el 80,27\%, de lo cual el saldo de esta se ha destinado en un 53,18 \% a cartera de consumo, seguido por un $35,82 \%$ a cartera de microcrédito, a crédito inmobiliario en un $6,48 \%$, y en un $4,12 \%$ a crédito comercial.

En cambio, la cartera bruta del s1 representa el $65,36 \%$ del total del sFPs, con un crecimiento del $10,84 \%$ compuesto anual, y son las más colocadoras las COAC: Juventud Ecuatoriana Progresista, Jardín Azuayo, Policía Nacional, 29 de octubre y Cooprogreso (representan el $46,15 \%$ de la cartera de este segmento y el 30,16\% del SFPS).

En cuanto a los pasivos de todas las organizaciones que son parte del SFPS, representan el 19,31\% del SFN y presentan un crecimiento del 8,18 \% compuesto anual en este mismo periodo, mientras que el SFN en su conjunto creció en un 1,04\% que contrasta con lo que sufrió la disminución de $0,47 \%$ de los bancos privados, por la disminución de las cuentas de obligaciones con el público, por más de USD 3181 millones, de manera que son los mayores afectados.

Las COAC del SFPS representan el 30,71\% de la participación de mercado, de las captaciones en depósitos a plazo fijo, de modo que el s1 representa el $67,86 \%$ con un crecimiento del $14,49 \%$ compuesto anual, y son las más captadoras las COAC:
Juventud Ecuatoriana Progresista, Jardín Azuayo, Cooprogreso, "29 de Octubre" y Policía Nacional, (representan el 47,71 \% de este segmento).

Las cooperativas de ahorro y crédito del SFPS representan el 12,85 \% de la participación de mercado de los depósitos a la vista del SFN, en el cual el s1 representa el 66,56 \% del total del SFPS, con un crecimiento de apenas un 7,09 \% compuesto anual, y son las más captadoras las COAC: Juventud Ecuatoriana Progresista, Jardín Azuayo, 29 de octubre, Policía Nacional y Riobamba (representan el 45,34 \% del segmento).

El patrimonio de las COAC representa el 26,68\% del SFN y presentan un crecimiento del $13,49 \%$ compuesto anual en este mismo periodo, de modo que el capital social representa el 50,86\% y las reservas el $37,70 \%$. El s1 representa el $61,36 \%$ a diciembre del 2015 de todo este sector, con un crecimiento compuesto del 13,92\%, del que son parte las COAC: Juventud Ecuatoriana Progresista, Policía Nacional, Jardín Azuayo, "29 de octubre" y San Francisco (representan el 43,53 \% del segmento).

Los resultados obtenidos de las organizaciones que son parte del sFPs presentan un modesto crecimiento del 2,80 \% compuesto anual en este periodo, que es, sin embargo, el doble registrado por el SFN (alcanzo el 1,29 \%) y el de los bancos privados (apenas el 0,56\%); sin embargo, de esto las coAC representan el 19,97 \% de participación de los resultados obtenidos por el SFN. Los resultados obtenidos por el s1 son más alentadores, pues con un porcentaje del 82,14\% de participación a diciembre del 2015 de todo el SFPs, presentan un crecimiento del 8,62 \%; en este segmento, se encuentran las COAC: Juventud Ecuatoriana Progresista, Jardín Azuayo, San Francisco, Cacpeco y Alianza del Valle (representan el $51 \%)$.

\section{Referencias}

Coraggio, J. L. (2011). Economía social y solidaria: el trabajo antes que el capital. Quito: Ediciones Abya-Yala.

Jones, G. R. y George, J. M. (2003). Administración contemporánea ( $4^{\mathrm{a}}$ ed.). México: McGraw Hill.

González, L., Arias, N. y San Bartolomé, J. C. (2012). El balance social cooperativo: una herramienta necesaria para la gestión cooperativa. Rosario: ACIAméricas. 
Horngren, C., Foster, G. y Datar, S. (2002). Contabilidad de costos un enfoque gerencial (10 ${ }^{\mathrm{a}}$ ed.). México: Prentice Hall.

Ley Orgánica de la Economía Popular y Solidaría y del Sector Financiero Popular y Solidario. Registro Oficial 444 de 10 de mayo de 2011.

Meigs, R. B. (1980). Principio de auditoría. México: Diana.

Palomo, R. J. y Mateu, J. L. (1999). Verificación de la aplicación del criterio de territorialidad. Ciriec-España, (32), 157-188.

Portus, L. (1997). Matemáticas financieras (4a ed.). Bogotá: McGraw-Hill.

Registro Oficial. (2014). Código Orgánico Monetario y Financiero. Ecuador: Registro Oficial.

Reglamento a la Ley Orgánica de la Economía Popular y Solidaría. Decreto N. ${ }^{\circ} 1061$. Registro Oficial. Suplemento 648 de 27 de febrero de 2012.
Resolución N. ${ }^{\circ}$ SEPS-IFPS-2012-0025 de 05 de diciembre de 2012 .

Richer, M. (2001, mayo). Economía social y solidaria. Intento de definición. Revista venezolana de economía social, 1-27.

Ross, S. A., Westerfield, R. W. y Jordan, B. D. (2001). Fundamentos de finanzas corporativas (5a ed.). México: McGraw-Hill.

Superintendencia de Economía Popular y Solidaria-seps. (2012, octubre). El Sector Económico Popular y Solidario en el Ecuador. Boletín de Coyuntura, 1, 13.

Superintendencia de Economía Popular y Solidaria-sePs. (2013, junio 30). Boletín financiero. Sector financiero popular y solidario (SFPS). Quito: SEPS.

Superintendencia de Economía Popular y Solidaria-SEPS. (2013, abril). Un vistazo del sector cooperativo por segmento y niveles. Quito: SEPS. 